\section{Phenotypic Characterization and Simple Sequence Repeat Identification of Red-fleshed Kiwifruit Germplasm Accessions}

\author{
Yan-Chang Wang ${ }^{1}$ and Lei Zhang \\ Key Laboratory of Plant Germplasm Enhancement and Speciality Agriculture, \\ Wuhan Botanical Garden, Chinese Academy of Sciences, Wuhan 430074, \\ China
}

\section{Yu-Ping Man}

School of Life Sciences, South-central University for Nationalities, Wuhan, 430074, China

\section{Zuo-Zhou Li}

Key Laboratory of Plant Germplasm Enhancement and Speciality Agriculture, Wuhan Botanical Garden, Chinese Academy of Sciences, Wuhan 430074, China

\section{Rui Qin}

School of Life Sciences, South-central University for Nationalities, Wuhan, 430074, China

Additional index words. Actinidia, red-flesh, SSR marker, phenotype, fruit character

\begin{abstract}
Big fruit size and nice red pigmentation combined with good flavor should be the major target for red-fleshed kiwifruit (Actinidia spp.) breeding programs. Genetic diversity and plant characteristics were evaluated on a set of kiwifruit accessions with predominantly red flesh to identify the superior individuals for further breeding or study of commercial application. The leading phenotypic characters varied widely among the accessions. Accession $R$ reached average fruit weight $\approx \mathbf{1 0 0} \mathrm{g}$, whereas it ranged from 43.15 to $84.71 \mathrm{~g}$ for the other accessions. Fruits of $L$ and $Q$ were flatter in shape than the others. The core volume accounted for fruit proportions ranging from $2.33 \%$ to $11.42 \%$. 'Chuhong', 'Honghua', and $\mathrm{K}$ exhibited a round fruit apex, whereas most others showed a depressed apex. $R, L$, and $Q$ had the highest $a^{*}$ values in the inner pericarp and also the most appealing visual coloration. Results revealed significantly higher soluble solid content (SSC), total sugar, and sugar/acid ratio in $Q$, $R$, and $L$. The 12 pairs of simple sequence repeat (SSR) markers were successfully used to characterize the genetic variability and confirm true-to-type identity for four accessions. However, the limited number of markers had no ability to discriminate among the other 11 accessions. Based on additional $28 \mathrm{SSRs}$, six of the indistinguishable accessions were confirmed to be genetically different, and three seemed to belong to the same clone vine. The results demonstrated that application of SSR data could improve the efficiency of identifying red-fleshed kiwifruit germplasm.
\end{abstract}

in the world, 'Hongyang', was developed and firstly released from Cangxi County of Sichuan Province in China (Wang, 2003; Wu, 1992), which now has the largest cultivation acreage of red-fleshed kiwifruit in China. Red-fleshed kiwifruit are novel fruits that have unique and attractive appeal and make consumers willing to buy, although the green- and yellow-fleshed cultivars still represent the main body of the kiwifruit traded internationally (Ferguson and Seal, 2008). To date, 'Hongyang' has been introduced into at least eight Provinces in China. Other cultivars or selections with red flesh or red core include 'Chuhong', 'Hongmei', 'Honghua', 'Yuanhong', 'Xiangjihong', 'Tianyuanhong', and 'Longshanhong', which were later registered in China (Wang et al., 2005a, 2005b, 2006; Wu and Wu, 1998). However, their comprehensive characteristics (especially the red coloration, fruit size, sweetness, or flavor) did not meet 'Hongyang' standards. However, as the predominant cultivar in redfleshed kiwifruit production $(\approx 5000$ ha in China), besides its small fruit size, 'Hongyang' is susceptible to Pseudomonas syringae pv. Actinidiae ( $P s a$ ), one of the most dangerous bacterial pathogens of kiwifruit plants worldwide, which causes devastating damage to phyllosphere organs (Balestra et al., 2009; Feng and Li, 2009; Rossetti and Balestra, 2008). Moreover, its fruit coloration is badly inhibited by high day temperatures, which results in an obvious reduction or almost disappearance in pigmentation of the inner pericarp (Zhong et al., 2007; unpublished data). Larger fruit, higher intensity of the red pigments, and their high stability under warmer climates combined with strong resistance to $P s a$ and good flavor should be the major targets for breeding and improvement of red-fleshed cultivars.

Kiwifruit cultivars on the world market are mostly selected from wild germplasm, budsports, seedlings, and controlled crosses (Chen et al., 2009; Ferguson and Huang, 2007). The kiwifruit industry in Cangxi of Sichuan Province began with a batch of mixed seeds of wild A. chinensis from the Funiu Mountains, Henan Province in 1978, from which 'Hongyang' was developed. Large variations in flesh color, size, shape, flavor compounds, storage life, and
Red flesh was first found in the fruit of A. chinensis from Hubei (Liang, 1982) and described by Cui (1993) again. In the late 1990s, the first red-fleshed kiwifruit cultivar

Received for publication 27 Oct. 2011. Accepted for publication 26 Mar. 2012.

This work was supported by the National Natural Science Foundation of China (Grant no. 30671433, 31171945), the cooperation project from the Government of Cangxi County in Sichuan Province, and the Knowledge Innovation Program (KSCX2YW-Z-052) of Chinese Academy of Sciences.

We thank Miss Zhan Qu for her kind review in language and thank Shi-Song He and Shi-Quan Wu for their help during the sampling.

${ }^{1}$ To whom reprint requests should be addressed; e-mail kiwifruit@wbgcas.cn.
Table 1. Accessions origins used in this study.

\begin{tabular}{|c|c|}
\hline $\begin{array}{l}\text { Cultivar name or code } \\
\text { of accession }\end{array}$ & Origins \\
\hline \multicolumn{2}{|l|}{ Cultivars } \\
\hline Hongyang & $\begin{array}{l}\text { One seedling from mixed wild-collected seeds of } A \text {. chinensis var. } \\
\text { chinensis and } A \text {. chinensis var. deliciosa from the Funiu Mountains } \\
(\mathrm{Wu}, 1992)\end{array}$ \\
\hline Chuhong & $\begin{array}{l}\text { One wild plant of } A \text {. chinensis var. chinensis from the mountainous } \\
\text { region of Xupu County in the western part of Hunan Province } \\
\text { (Wang et al., 2005b) }\end{array}$ \\
\hline Hongmei & $\begin{array}{l}\text { One seedling from wild-collected seeds of } A \text {. chinensis var. deliciosa in } \\
\text { the northern mountains of the Sichuan Basin (Wang et al., 2005a) }\end{array}$ \\
\hline Honghua & $\begin{array}{l}\text { One hybrid of 'Hongyang' and } A \text {. chinensis var. deliciosa male plant } \\
\text { (Wang et al., 2006) }\end{array}$ \\
\hline \multicolumn{2}{|l|}{ Accessions } \\
\hline $\mathrm{AB}, \mathrm{AF}, \mathrm{H}, \mathrm{K}, \mathrm{L}, \mathrm{O}, \mathrm{Q}, \mathrm{R}$ & $\begin{array}{l}\text { Selections from the seedlings of mixed wild-collected seeds of } \\
\text { A. chinensis var. chinensis and A. chinensis var. deliciosa from the } \\
\text { Funiu Mountains }\end{array}$ \\
\hline $\mathrm{C}, \mathrm{I}, \mathrm{J}$ & Phenotypic variations from 'Hongyang' orchards \\
\hline
\end{tabular}


Breeding, Cultivars, Rootstocks, and Germplasm Resources

other fruit characteristics have been increasingly discovered in the seedling population that was derived from the batch of wild seeds. Some of the fruiting individuals were wrongly introduced to 'Hongyang' orchards in Cangxi or other provinces. However, few organized investigations have been conducted to select for 'Hongyang' purification and cultivar improvement in the seedling population because the seeds were originally distributed among some local farmers.

In the present study, some red-fleshed individual plants with great phenotypic variations from the historic seedling population were selected, and their vegetative and fruiting traits were investigated under the same conditions within the same garden. They were characterized to identify better parents for a breeding program of red-fleshed kiwifruit or further evaluate the commercial potential. In addition, novel variations can be derived from the budsports of 'Hongyang' and improper introduction. SSR markers were used to characterize genetic variability and confirm true-totype identity of the accessions.

\section{Materials and Methods}

Plant material. A large seedling population was produced for rootstocks in Cangxi County in 1982 using the wild kiwifruit seeds (A. chinensis var. chinensis and A. chinensis var. deliciosa) from the Funiu Mountains. Based on a great deal of investigations of the ungrafted seedlings, hundreds of superior individuals were collected for further identification for their use in the red-fleshed kiwifruit breeding program. Eight red-fleshed accessions (AB, AF, H, K, L, O, Q, and R) were selected from the superior individuals. Accessions C, I, and J were the three phenotypic variations in 'Hongyang' commercial orchards. Mature shoots of these accessions were grafted on seedling rootstocks in a repository at Cangxi in 1999. Each of the variants was propagated by grafting (i.e., three to five plants) and managed according to normal commercial practice. 'Hongyang', 'Honghua', 'Hongmei', and 'Chuhong' were grafted in parallel and managed together with other 11 accessions in the repository and used as controls for plant evaluation and microsatellite analysis. The origins of the materials are listed in Table 1.

Evaluation of fruit characteristics. Fruit shape, hairs, and fruit apex shape were described quantitatively. From 2006 to 2007, eight to 10 randomly selected fruits of above females were harvested from each vine at 150 to $155 \mathrm{~d}$ after flowering, when the fruit was considered ripe for commercial picking, and used for evaluating shape, color, and hair characteristics. These are the traits typically assessed for commercial desirability. Fruit apex shape was divided into eight types according to $\mathrm{Hu}$ et al. (2006) and UPOV (2003) guidelines for Actinidia. Individual average fruit weight, longitudinal diameter (LD), maximum transverse diameter $\left(\mathrm{TD}_{\max }\right)$, and minimum transverse diameter $\left(\mathrm{TD}_{\min }\right)$ of fruit at the equator were measured at harvest stage.

Table 2. Fruit weight, size, shape, and core volume of Actinidia accessions.

\begin{tabular}{lllcccc}
\hline Accessions & $\mathrm{Wt}(\mathrm{g})$ & $\mathrm{LD}(\mathrm{mm})$ & $\mathrm{TD}_{\max }(\mathrm{mm})$ & $\mathrm{TD}_{\min }(\mathrm{mm})$ & $\mathrm{TI}$ & $\mathrm{PCV}(\%)$ \\
\hline $\mathrm{AB}$ & 63.66 & 43.5 & 44.89 & $42.5^{*}$ & 0.9468 & 8.12 \\
$\mathrm{AF}$ & 59.68 & 52.63 & 44.32 & 40.95 & 0.9240 & 6.45 \\
$\mathrm{C}$ & 43.15 & 41.34 & 40.06 & 38.58 & 0.9631 & 5.34 \\
$\mathrm{H}$ & 54.75 & 50.04 & 44 & 38.15 & 0.8670 & 7.25 \\
$\mathrm{I}$ & 57.49 & 54.21 & 43 & 38.43 & 0.8937 & 2.33 \\
$\mathrm{~J}$ & $78.12^{*}$ & 58.52 & $46.53^{*}$ & 42.82 & 0.9203 & 6.71 \\
$\mathrm{~K}$ & $84.59^{*}$ & $63.15^{*}$ & $47.85^{*}$ & 43.5 & 0.9091 & 7.8 \\
$\mathrm{~L}$ & $82.97^{*}$ & 58.06 & $50.78^{*}$ & $41.6^{*}$ & 0.8192 & $11.42^{* *}$ \\
$\mathrm{O}$ & $84.71^{*}$ & $67.27^{*}$ & $46.5^{*}$ & $42.15^{*}$ & 0.9065 & 6.59 \\
$\mathrm{Q}$ & 70.08 & 57.25 & 45.79 & 38.7 & 0.8452 & $9.89^{*}$ \\
$\mathrm{R}$ & $100.82^{* *}$ & $66.26^{*}$ & $50.29^{*}$ & $43.19^{*}$ & 0.8588 & $9.1^{*}$ \\
Honghua & $81.13^{*}$ & $65.97^{*}$ & 44.73 & 40.82 & 0.9126 & 4.65 \\
Hongmei & 61.68 & 55.1 & 41.33 & 39.03 & 0.9444 & 6.68 \\
Hongyang & 68.5 & 54.74 & 43.67 & 40.44 & 0.9260 & $9.09^{*}$ \\
\hline
\end{tabular}

The value is an average of 24 to 50 fruits per accession. *a $<0.05, * * a<0.01$ (Student's $t$ test).

$\mathrm{LD}=$ longitudinal diameter; $\mathrm{TD}_{\max }=$ maximum transverse diameter; $\mathrm{TD}_{\min }=$ minimum transverse diameter; $\mathrm{TI}=$ transverse diameter index $\left(=\mathrm{TD}_{\min } / \mathrm{TD}_{\max }\right) ; \mathrm{PCV}=$ percentage of core volume $\left[=\left(\mathrm{ATDC}^{2}\right.\right.$ $\left.\left.\mathrm{TD}_{\text {mean }}^{2}\right) \times 100 \%\right]$; ATDC $=$ average transverse diameter of core.

Table 3. Descriptions of fruit shape, hair character, and stylar end shape of Actinidia accessions.

\begin{tabular}{|c|c|c|c|}
\hline Accessions & Fruit shape & Hair & Shape of stylar end \\
\hline $\mathrm{AB}$ & Oblate short cylindrical & Absent & Depressed, then blunt protruding \\
\hline $\mathrm{AF}$ & Oblate conical & Absent & Depressed, then blunt protruding \\
\hline $\mathrm{C}$ & Oblate short cylindrical & Sparse, velutinous & Deeply depressed \\
\hline $\mathrm{H}$ & Oblate short cylindrical & Absent & Depressed \\
\hline I & Oblate long cylindrical & Absent & Depressed \\
\hline $\mathrm{J}$ & Oblate cylindrical & Sparse, velutinous & Narrow, depressed \\
\hline $\mathrm{K}$ & Oblate cylindrical & Sparse, velutinous & Rounded \\
\hline $\mathrm{L}$ & Oblate cylindrical & Absent & Depressed, then blunt protruding \\
\hline $\mathrm{O}$ & Oblate cylindrical & Sparse, velutinous & Rounded \\
\hline Q & Oblate conical & Sparse, velutinous & Depressed, then blunt protruding \\
\hline $\mathrm{R}$ & Oblate cylindrical & Absent & Depressed, then blunt protruding \\
\hline Honghua & Oblate cylindrical & Velutinous & Rounded \\
\hline Hongmei & Cylindrical & Absent & Depressed \\
\hline Hongyang & Oblate short cylindrical & Sparse, velutinous & Deeply depressed \\
\hline Hongyang & Oblate short cylindrical & Sparse, velutinous & Deeply depressed \\
\hline
\end{tabular}
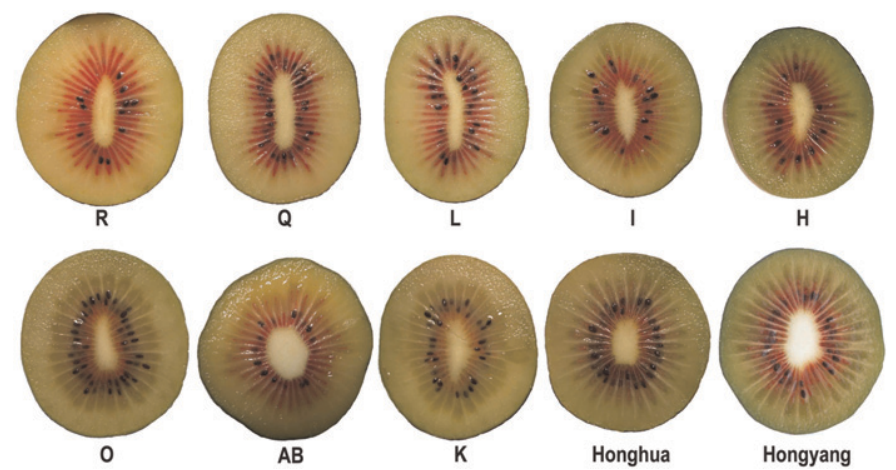

Fig. 1. Coloration in inner pericarp of some red-fleshed kiwifruit accessions. The fruits were harvested at 150 to $155 \mathrm{DAF}$ and then stored at $4{ }^{\circ} \mathrm{C}$ for 1 month before photographs were taken. $\mathrm{DAF}=$ days after flowering.

Fruit core maximum and minimum transverse diameter were also measured for calculating the core average transverse diameter (ATDC).

Fruits were cut at the equatorial region for color measurements. The color of two exposed section planes of the inner pericarp flesh were assessed using a Minolta CR-300 chroma meter (Osaka, Japan) with a measuring head, diffuse illumination, and $0^{\circ}$ viewing angle geometry. These parameters provided an $8-\mathrm{mm}$ diameter measuring area for each specimen. Channel 00 of the chroma meter was first calibrated to a white calibration plate. The L*-a*-b* color space (CIELAB) (CIE, 1976) (Pointer, 1981) was chosen for the present study, where $\mathrm{L}^{*}$ indicates lightness and $-a^{*}-b^{*}$ are the chromaticity coordinates. $\mathrm{L}^{*},-\mathrm{a}^{*}$, and $-\mathrm{b}^{*}$ range over a scale from 0 for black to 100 for white, -50 for green to 50 for red, and -50 for blue to 50 for yellow, respectively. Measurements were conducted at six different sites on the two exposed section planes, and the average was calculated for each fruit. For each measurement, the center of the measurement head was put just at the 


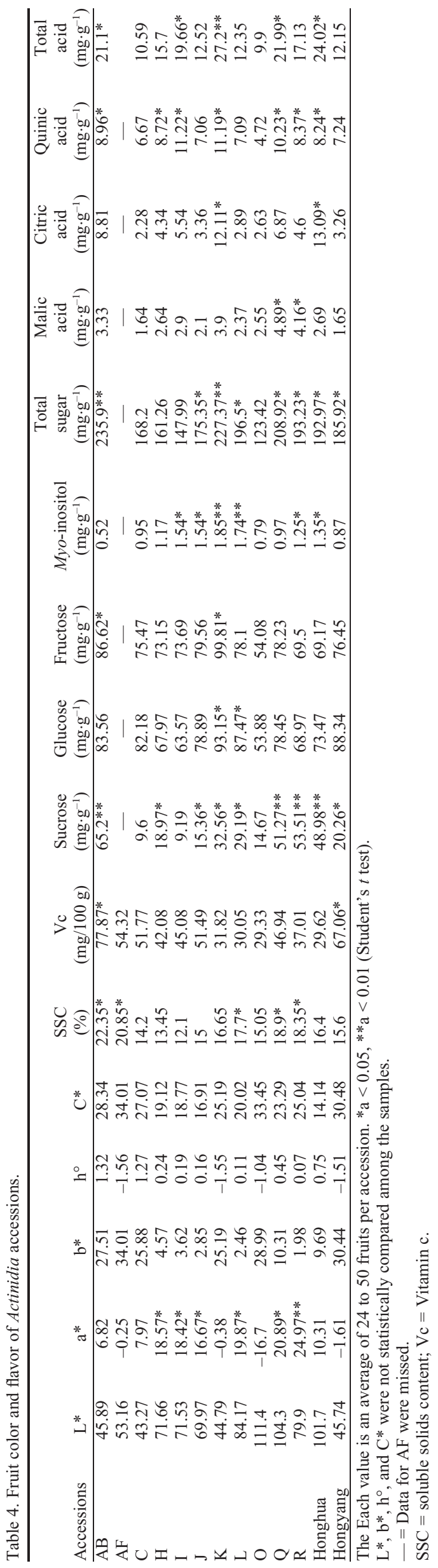

locule tissues. Finally, $\mathrm{h}^{\circ}$ (hue angle) and $\mathrm{C}^{*}$ were calculated using the equations subsequently (Stintzing et al., 2003).

$$
\begin{gathered}
\mathrm{h}^{\circ}=\arctan \left(\mathrm{b}^{*} / \mathrm{a}^{*}\right) \\
\mathrm{C}_{\mathrm{ab}}^{*}=\sqrt{a^{* 2}+b^{* 2}}
\end{gathered}
$$

Soluble solid content was measured by squeezing juice drops from the inner pericarp flesh from the two exposed planes of the equatorial region into an Atago PR-32 refractometer (Tokyo, Japan) and recording the SSC.

Fruit vitamin $\mathrm{C}$ content was determined using an ultraviolet-spectrophotometer (Zhang, 2010). Initially, the standard vitamin $\mathrm{C}$ solutions were scanned between 200 and $300 \mathrm{~nm}$, and an absorption peak set at $243 \mathrm{~nm}$ was used to construct the calibration curve. Five fruits were collected from each vine and washed, wiped dry, and weighed. Two grams of flesh were collected from the inner pericarp of each fruit and ground to a fine powder in liquid nitrogen, resuspended in $750 \mu \mathrm{L} 3 \mathrm{M}$ $\mathrm{HCl}$, and mixed with $750 \mu \mathrm{L} \mathrm{ddH}_{2} \mathrm{O}$ in a 2.0-mL tube. The extracts were subsequently centrifuged at $15,294 \mathrm{~g}$ at $4{ }^{\circ} \mathrm{C}$ for $15 \mathrm{~min}$, and $200 \mu \mathrm{L}$ of the supernatant was transferred

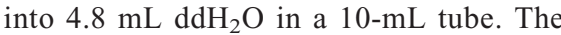
mixture was used to measure vitamin $\mathrm{C}$ concentration. Vitamin $\mathrm{C}$ content was expressed as milligrams of ascorbic acid per $100 \mathrm{~g}$ of fresh tissue.

Extraction and derivatization of inner pericarp sugars, sugar alcohols, and organic acids followed the protocol initially developed in sweet orange with minor modifications (Kelebek et al., 2009). Three grams of flesh were collected from five individual fruits for each sample and homogenized with $10 \mathrm{~mL}$ of $80 \%$ methanol (v/v). The homogenate was incubated for $15 \mathrm{~min}$ in a water bath maintained at $75{ }^{\circ} \mathrm{C}$, cooled to room temperature to sonicate for $45 \mathrm{~min}$, then centrifuged at $4000 \mathrm{~g}$ for $10 \mathrm{~min}$ at $20^{\circ} \mathrm{C}$. Precipitate was extracted twice with $80 \%$ methanol $(\mathrm{v} / \mathrm{v})$ using the described procedure. The supernatants were pooled, freeze-dried, and sequentially derivatized with hydroxylamine hydrochloride, hexamethyldisilazane, and trimethylchlorosilane. After derivatization, metabolites were analyzed with an Agilent 6890N GC (Agilent Technology, Palo Alto, CA). Injection of a $1-\mu \mathrm{L}$ sample was performed at $270{ }^{\circ} \mathrm{C}$ in splitless mode with $\mathrm{N}_{2}$ carrier gas flow set to $1 \mathrm{~mL} \cdot \mathrm{min}^{-1}$. Chromatography was performed using an HP-5 capillary column $(30 \mathrm{~m} \times 0.25 \mathrm{~mm} \times 0.1 \mu \mathrm{M})$. The temperature program was initiated with an isothermal at $130{ }^{\circ} \mathrm{C}$ followed by $8{ }^{\circ} \mathrm{C} \cdot \mathrm{min}^{-1}$ to $152^{\circ} \mathrm{C}, 12^{\circ} \mathrm{C} \cdot \mathrm{min}^{-1}$ to $176^{\circ} \mathrm{C}, 20^{\circ} \mathrm{C} \cdot \mathrm{min}^{-1}$ to $238{ }^{\circ} \mathrm{C}, 24^{\circ} \mathrm{C} \cdot \mathrm{min}^{-1}$ to $280{ }^{\circ} \mathrm{C}$, and a final 4 min heating at $280^{\circ} \mathrm{C}$. Sugars, sugar alcohols, and organic acids were quantified based on standard curves generated for each metabolite and internal standard. All chemical standards were obtained from Sigma-Aldrich (St. Louis, MO). Mean values were calculated for each different character measurement and used for analyses. 
Table 5. SSR primer pairs and the allele numbers that they amplified in the accessions.

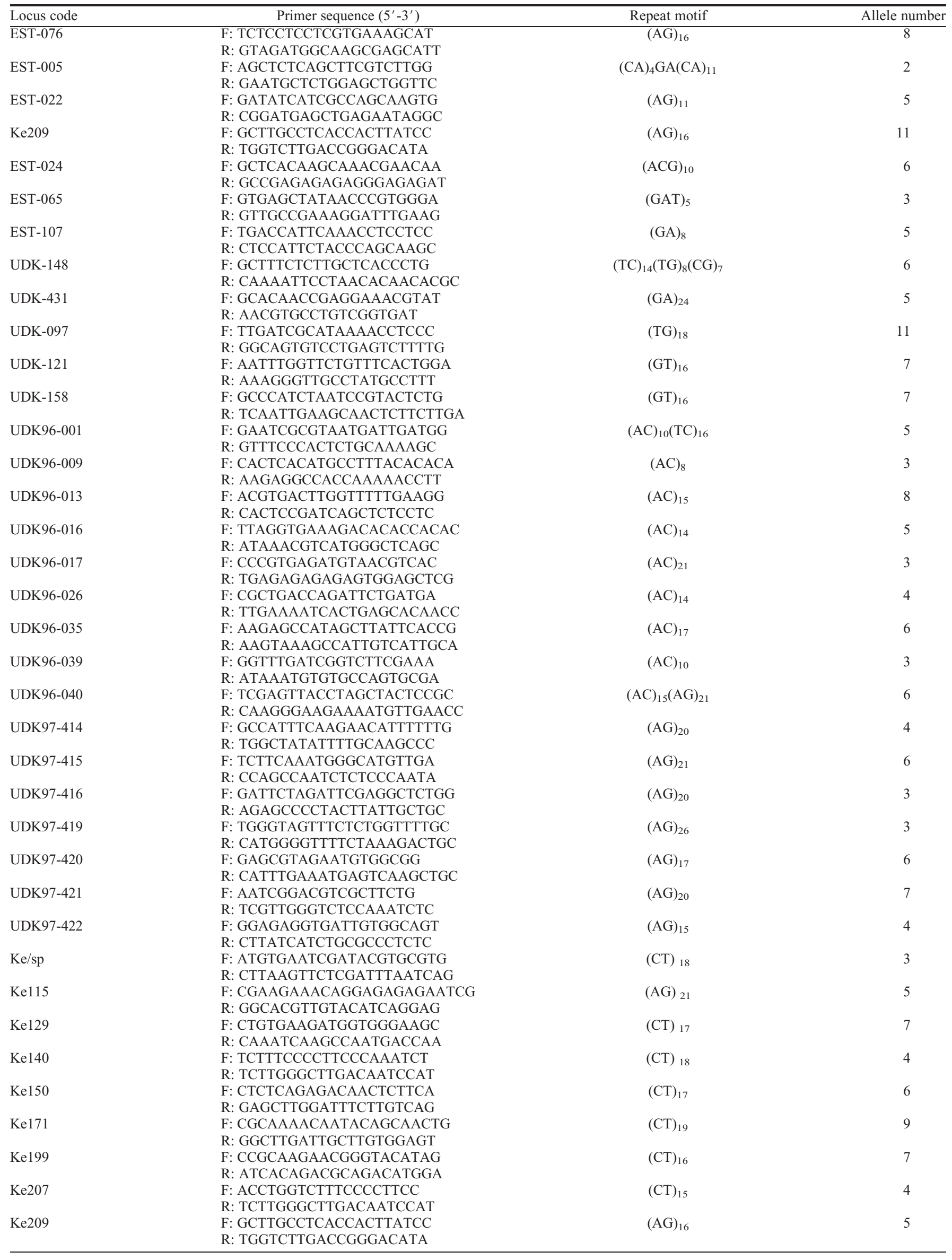


Table 5. (Continued) SSR primer pairs and the allele numbers that they amplified in the accessions.

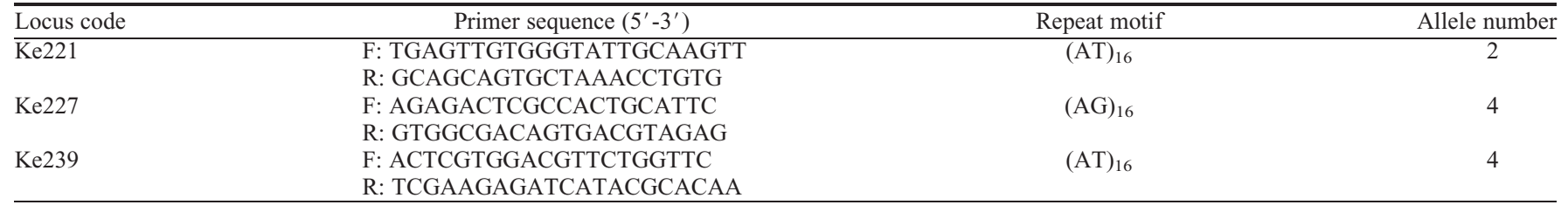

From EST-076 to UDK-158, 12 SSR primer pairs were used at the beginning. Additional 28 markers were indicated as those from UDK96-001 to Ke239. $\mathrm{SSR}=$ simple sequence repeat.

DNA extraction and microsatellite analysis. Total genomic DNA was isolated from fresh leaves using a modification of the CTAB procedure reported by Doyle and Doyle (1987). At the beginning, 12 SSR primer pairs (Table 4) were selected to evaluate the level of polymorphism among kiwi cultivars following a preliminary screening of 40 genomic SSR primer pairs. In the additional experiments, more than 50 SSR primer pairs were used for screening and then 28 SSR primer pairs were adopted for final identification. Polymerase chain reactions were performed using a protocol described by Zhang (2010) with minor modifications. The reaction mixtures contained the following in a total $10 \mu \mathrm{L}$ volume: $50 \mathrm{ng}$ genomic DNA, $1 \mu \mathrm{L} 10 \times$ buffer $(10 \mathrm{~mm}$ Tris$\mathrm{HCl}, \mathrm{pH}$ 8.3, $500 \mathrm{~mm} \mathrm{KCl}), 1.5 \mathrm{~mm} \mathrm{MgCl}_{2}, 0.1$ mM of each primer, $0.2 \mathrm{~mm} \mathrm{dNTPs}$, and $0.5 \mathrm{U}$ of Taq DNA polymerase. The amplification was carried out on a Master Gradient (Eppendorf, Germany) thermal cycler using the following temperature profile: $95^{\circ} \mathrm{C}$ for $5 \mathrm{~min}$ followed by six cycles of $94^{\circ} \mathrm{C}$ for $50 \mathrm{~s}, 61^{\circ} \mathrm{C}$ (decreased by $1{ }^{\circ} \mathrm{C}$ at each cycle) for $50 \mathrm{~s}, 72^{\circ} \mathrm{C}$ for $1 \mathrm{~min}$ followed by 30 cycles of $94{ }^{\circ} \mathrm{C}$ for $50 \mathrm{~s}, 56{ }^{\circ} \mathrm{C}$ for $50 \mathrm{~s}, 72^{\circ} \mathrm{C}$ for $1 \mathrm{~min}$ and a final $72{ }^{\circ} \mathrm{C}$ for 8 $\mathrm{min}$. The amplification products were added to $7.5 \mu \mathrm{L}$ loading buffer ( $98 \%$ formamide, $0.05 \%$ bromophenol blue, $0.05 \%$ xylene cyanol, and $10 \mathrm{~mm} \mathrm{NaOH}$ ), denatured at $95{ }^{\circ} \mathrm{C}$ for $5 \mathrm{~min}$, and separated in a $6 \%$ denaturing polyacrylamide gel using a Sequi-Gen GT/Power Pac 3000 Sequencer System (Bio-Rad) for 1 to $1.5 \mathrm{~h}$ at $55 \mathrm{~W}$. Silver staining was conducted according to Sanguinetti et al. (1994) with slight modifications. A 25-bp DNA ladder (Promega, Madison, WI) was used as the size standard, and bands were scored as $1,2,3 \ldots$ from the largest to the smallest size following the scoring code at each locus to represent SSR alleles.

Data analysis. The proportion of core volumes in one fruit was estimated by treating all kiwifruits as approximate cylinders. Some parameters were calculated as follows:

1) Percentage core volume: $\mathrm{PCV}=\left(\mathrm{ATDC}^{2} /\right.$ $\left.\mathrm{TD}_{\text {mean }}^{2}\right) \times 100 \%$;

2) Transverse diameter index: $\mathrm{TI}=\mathrm{TD}_{\min } /$ $\mathrm{TD}_{\max }(\mathrm{Wu}$ et al., 2011).

Fruit morphology and fruit flavor among the accessions were compared by analysis of variance using SAS software (Version 9.1; SAS Institute Inc., Cary, NC) for Windows. Student-Newman-Keuls test was applied during the analysis at the $P \leq 0.05$ or 0.01 level of significance.
The original molecular data were transformed to a binary data matrix if some of the samples were polyploid, and each SSR locus allele was recorded as 0 if the allele was absent in the progeny and 1 if present (Mengoni et al., 2000). Dice similarity coefficients among the collected plants were calculated for each locus, and then the average similarity coefficients over all loci were used for clustering. Clustering analyses were performed using the unweighted pair group method with arithmetic averages (UPGMA) in the NTSYS-pc 2.1 package (Rohlf, 2000). Principal component analysis (PCA) was performed using SAS software (Version 9.1; SAS Institute Inc.) for Windows.

\section{Results}

Fruit morphology. Red-fleshed R had significantly bigger fruit among these accessions and cultivars with average weight $\approx 100 \mathrm{~g}$. Fruit weight of J, K, L, O, and 'Honghua' ranged from $78.12 \mathrm{~g}$ to $84.71 \mathrm{~g}$, which was significantly heavier than the other samples. Fruit size of L was quite distinct from that of its accessions' maternal plant R. Among those accessions with heavier fruits, both the LD and the $\mathrm{TD}_{\max }$ of $\mathrm{K}, \mathrm{L}, \mathrm{O}$, and R were significantly longer than those with lighter fruits. However, increase in fruit weight of $\mathrm{J}$ and $\mathrm{L}$ was the result of an increase in fruit transverse diameter, not the longitudinal diameter (Table 2).

Generally, few kiwifruits had regular spheres. Based on data of all present samples, the overall mean value of TI was 0.9026 , which explained why the fruits looked flat. L, $\mathrm{Q}, \mathrm{R}$, and $\mathrm{H}$ had flatter fruit than the others because they had smaller TI $(\leq 0.8670)$. L had the largest core among all the samples; then Q, $\mathrm{R}$, and 'Hongyang', which had higher percentages of core volume (PCV) than 9\% (Table 2). Accession I showed a core with the smallest fruit volume $(\mathrm{PCV}=2.33 \%)$. The majority of the other accessions exhibited cylindrically shaped fruits as a whole (Table 3), but they were not an exact circular cylinder because the two ends of almost all fruits were not the same size. The fruit shoulder at the stalk end of 'Hongyang' was larger than the stylar end.

The fruit apex shape in kiwifruit is an easily distinguishable trait. Among red accessions, fruits of $\mathrm{K}, \mathrm{O}$, 'Chuhong', and 'Honghua' had a round fruit apex; however, many others exhibited a depressed stylar end (Table 3). Other differences were also observed, including diverse types of protrusions in the center of a depressed apex and altered volumes in the apex depression. The depression volume of 'Hongyang' was much greater than J, L, Q, R, AB, and AF (Table 3).

Fruit pigmentation in the inner paricarp and flavor characteristics. CIELAB is a onecolor system that takes into account all aspects of the color described by $\mathrm{L}^{*}, \mathrm{a}^{*}$, and $b^{*}$ parameters. The $a^{*}$ negative and positive are for green and red, respectively. Anthocyanin concentration in the inner pericarp of 'Hongyang' was significantly positively correlated with $\mathrm{a}^{*}$, indicated by a high correlation coefficient (unpublished data). Accession R had the highest $\mathrm{a}^{*}$ among the samples followed by Q, L, H, I, and J (Table 4). Consistently, the pericarp color in $\mathrm{R}, \mathrm{L}, \mathrm{I}, \mathrm{H}$, and Q was visually more appealing than other samples (Fig. 1). Two chroma parameters, $\mathrm{C}^{*}$ and $\mathrm{h}^{\circ}$, were derived from $a^{*}$ and $b^{*}$ and used to describe saturation of the color, a measure of how far the color is from the gray tone. The higher the $\mathrm{C}^{*}$ value, the more saturated the color. The hue angle parameter $\left(\mathrm{h}^{\circ}\right)$ describes the hue of the color, i.e., color tonalities. Based on the $\mathrm{h}^{\circ}$ measured, all the accessions and cultivars were the red type (Table 4).

$\mathrm{L}, \mathrm{Q}, \mathrm{R}, \mathrm{AB}$, and AF had significantly higher SSC than other samples, and the descending order was $\mathrm{AB}>\mathrm{AF}>\mathrm{Q}>\mathrm{R}>\mathrm{L}$ $>$ in other samples (Table 4). Fruit Vitamin c (Vc) content ranged from 29.33 to $77.87 \mathrm{mg}$ per $100 \mathrm{~g}$ fresh weight. Higher Vc content was detected in $\mathrm{AB}$ and 'Hongyang' compared with the other accessions (Table 4). Glucose and fructose were the two principle sugars in all samples. Sucrose was present but at a notably lower level. However, in Q, $\mathrm{R}, \mathrm{AB}$, and 'Honghua', it was detected at a slightly lower concentration than glucose or fructose. The sucrose concentrations corresponded closely to one-third to one-ninth of glucose or fructose for most other samples (Table 4). Fruits of $\mathrm{K}$ and $\mathrm{L}$ contained the highest concentration of myo-inositol followed by I, J, R, and 'Honghua'. The lowest concentrations of myo-inositol were detected in the remaining samples (Table 4). Quinic and citric acid were the two principal fruit acids. A comparison of total organic acids determined that $\mathrm{K}$ had significantly higher organic acid content among all samples, and 'Honghua', Q, AB, and I were the next highest among the accessions. Fruits of 'Hongyang' and $\mathrm{L}$ exhibited the highest sugar/acid ratio (Table 3).

Simple sequence repeat marker confirmation and principal component analysis. The number of alleles per locus of 12 polymorphic expressed sequence tag-SSR loci ranged from two to 11 with the average of 6.33 per locus in the 
present study (Table 5). A total of 76 alleles were identified from those SSR primers in the 15 samples. Among loci, Ke209 and UDK-097 exhibited the two highest levels of polymorphism with 11 alleles each, and EST-005 was least polymorphic with two alleles. The genetic similarities among accessions were evaluated by recording all alleles for UPGMA cluster analysis. Results showed that all the accessions clustered in two main groups (Fig. 2). One group included 'Chuhong' and 'Hongmei'; however, the two cultivars seemed to be genetically distant to each other (Fig. 2). The other group was subdivided into two subgroups; one subgroup included 'Honghua', $\mathrm{H}$, 'Hongyang', and four accessions (AF, C, I, and $\mathrm{J}$ ) and the other subgroup included $\mathrm{K}, \mathrm{O}$, and other four accessions (AB, L, Q, and R). However, each of the accessions, AF, C, I, and $\mathrm{J}$, was not distinguishable from the cultivar Hongyang. In addition, genetic differences were not found among $\mathrm{AB}, \mathrm{L}, \mathrm{Q}$, and $\mathrm{R}$ and between $\mathrm{K}$ and $\mathrm{O}$ (Fig. 2).

Principal component analysis was carried out using data of fruit characters and the 76 alleles of SSR loci. The first three principal coordinates accounted for $35.31 \%, 28.29 \%$, and $13.64 \%$, respectively, of the total variation of fruit (Fig. 3A-B). For the SSR variation, however, they accounted for $38.19 \%, 23.60 \%$, and $18.63 \%$, respectively. According to PCA results, no obviously different groups were observed in the Prin 1 - Prin2 plot (Fig. 3A) and Prin 1 - Prin3 plot (Fig. 3B) derived from fruit characters. However, 'Chuhong' and 'Hongmei' were separated from 11 accessions and two other cultivars based on Prin 1Prin2 plot (Fig. 3C) derived from SSR data. Based on the Prin 1 - Prin3 plot (Fig. 3D) derived from SSR data, the red-fleshed accessions were also classified into two groups, which is consistent with the UPGMA cluster analysis.

\section{Discussion}

To develop superior red-fleshed cultivars, we evaluated the new collections from the seedlings of mixed wild-collected seeds and the originally commercialized orchards of red-fleshed 'Hongyang' based primarily on phenotypic data. Many variations of the vegetative traits were observed among the accessions. In the present study, we focused on evaluation of fruit traits, which are even more directly related to cultivar improvement. TI (minimum transverse diameter/maximum transverse diameter) was adopted for describing the shape of kiwifruit, which was calculated according to the method described by $\mathrm{Wu}$ et al. (2011). However, the reverse ratio of greater equatorial diameter and lesser equatorial diameter were also adopted by the other researchers (Rashidi and Gholami, 2008; Zhu et al., 2002), which made the parameters of fruit symmetry higher than 1.0. Fruit symmetry (Zhu et al., 2002) and ellipsoid ratio (Rashidi and Gholami, 2008) were defined, respectively, in the two reports. It was suggested that the constant method should be adopted by different researchers when calculating the two

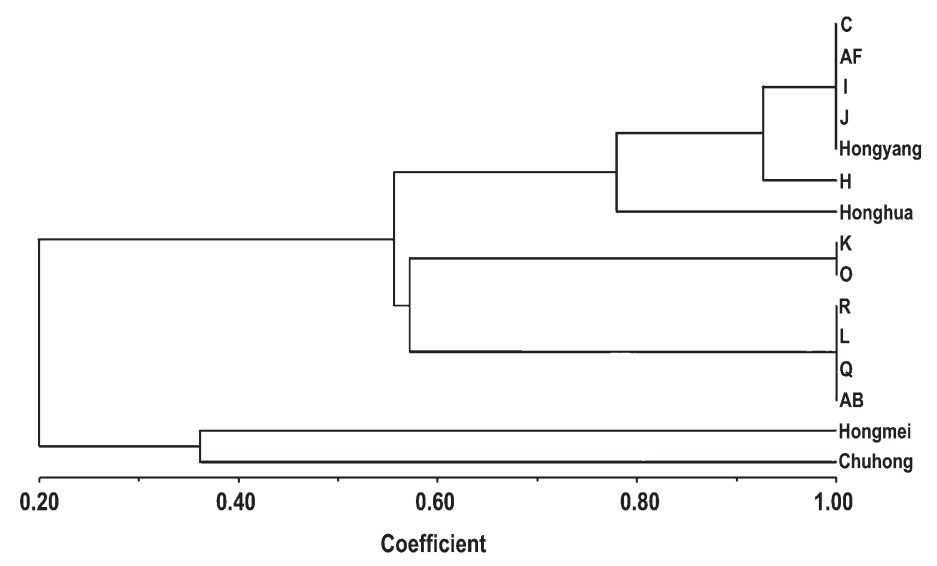

Fig. 2. UPGMA dendrogram using dice coefficient of genetic similarity among kiwifruit accessions based on the 12 SSR data. $\mathrm{C}, \mathrm{H}, \mathrm{I}, \mathrm{J}, \mathrm{K}, \mathrm{L}, \mathrm{O}, \mathrm{Q}, \mathrm{R}, \mathrm{AB}$, and AF were codes of the accessions. UPGMA = unweighted pair group method with arithmetic averages.
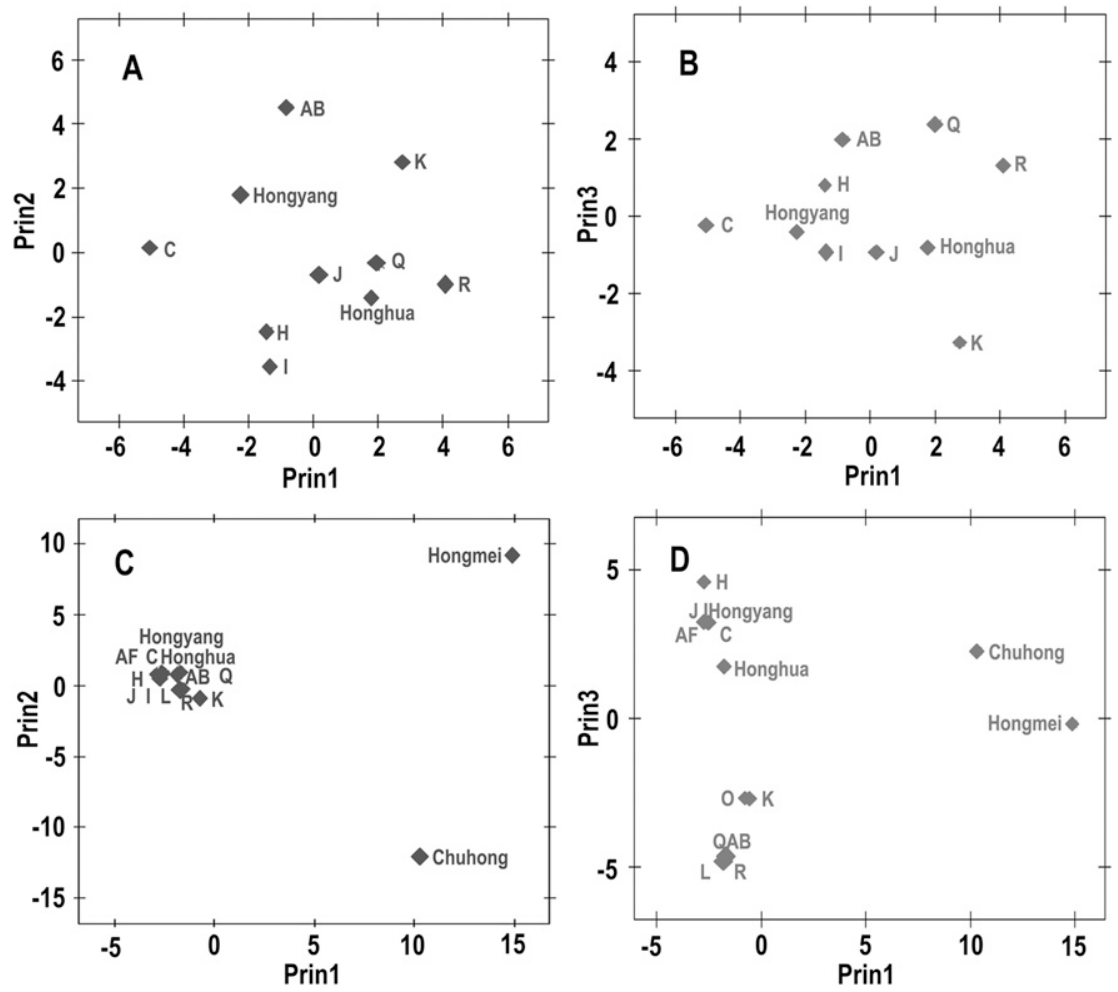

Fig. 3. Distribution of kiwifruit accessions on the principal component 1 (Prin1)-principal component 2 (Prin2) plot and principal component 1 (Prin1) - principal component 3 (Prin3) inferred from data of fruit characters (A-B) and SSR alleles (C-D). 'Chuhong', 'Hongmei', and AF were removed in Prin 1Prin2 plot (A) and Prin 1 - Prin 3 plot $(\mathbf{B})$ because of data missing of some fruit characters. SSR $=$ simple sequence repeat.

important parameters for describing the shape of kiwifruit, transverse diameter index and fruit elongation index, respectively, so that the data from different studies could be compared easily. Fruit core proportion is an important index to assess inner and middle pericarp quality (superior or inferior quality). Although traits including area, equatorial diameter, eccentricity, and core size (core width/ fruit width) have also been used to evaluate the fruit body or core volume, these parameters have lacked comprehensive and/or precise information for describing kiwifruit (Amodio et al., 2007; Zhu et al., 2002). PCV was first used in this study because the fruits could be treated as approximate cylinders.

The kiwifruit apex has been described as the stylar end by some researchers (Hassall et al., 1998; UPOV, 2003; White, 1986). The fruit apex in kiwifruit is probably derived during development from the outer pericarp of the multicarpellate ovary, part of the style or the ovary-stylar junction (Hopping, 1976; Hopping and Jerram, 1979; Howpage et al., 1998). Based on the present and unpublished data from hybrids of controlled crosses, kiwifruit 
apex morphology is seldom affected by environmental and cultivation factors, so genetic factors must be the primary determinants (Zhang, 2010). Fruit apex shape might be a convenient feature for distinguishing accessions. L, Q, and $\mathrm{R}$ exhibit a protrusion against the depressed surface. 'Hongyang', 'Hongmei', $\mathrm{H}, \mathrm{I}$, and $\mathrm{J}$ have a regular and wide depression, but 'Hort16A' has a beak (Muggleston et al., 1998; Wang et al., 2005b).

Our results allowed us to discriminate $\mathrm{L}$, $\mathrm{Q}, \mathrm{R}$, and $\mathrm{AB}$ with higher SSC and total sugar content from the other accessions. Compared with Actinidia arguta in which the predominant sugar of its fruit is sucrose, the main soluble sugars in the fruit of the present Actinidia chinensis accessions were glucose and fructose. In addition, $\mathrm{L}, \mathrm{Q}$, and $\mathrm{R}$ exhibited the deepest red color in the inner pericarp. A positive relationship between sugar concentration and pigmentation has been found in several plant species (Hiratsuka et al., 2001; Kawabata et al., 1999; Pirie and Mullins, 1977). High sugar concentration probably activates the sugar substrate for characteristic glycosylation, a key step in increasing red coloration in red-fleshed kiwifruit (Montefiori et al., 2010). In our results, L also exhibited the highest ratio of total sugar/total acid among the four accessions. Therefore, $\mathrm{L}$ is tentatively identified as a superior selection with combined fruit morphology and flavor characters.

Based on the introduction history of kiwifruit resources to Cangxi of Sichuan Province, most of the materials used for selection and cultivation were originally harvested from North China with the exception that only a few were collected from the northern mountains of the Sichuan Basin (Wang, 2003, 2005a, 2005b, 2006; Wu and $\mathrm{Wu}, 1998$; Zhu and $\mathrm{Li}, 1993)$. All the accessions except 'Chuhong' and 'Hongmei' were offsprings of wild individuals that originated from the same or closely related wild populations in the Funiu Mountains (in Central to North China). These accessions also had similar traits to A. chinensis var. chinensis. 'Hongmei' and 'Chuhong' were genetically distant to others probably because 'Hongmei' was derived from $A$. chinensis var. deliciosa and 'Chuhong' was developed from the mountainous region of western Hunan, which was far from Funiu Mountains and a natural hybrid zone between $A$. chinensis var. chinensis and A. chinensis var. deliciosa (Liao, 2011; Liu et al., 2010). The clustering results were also provided by the PCA using SSR data.

Several researchers have conducted molecular characterization of kiwifruit germplasm. Nine SSR markers were used to identify 48 cultivars and selections (Zhen et al., 2004) and the single SSR locus UDK96-414 successfully differentiated 47 genotypes. Discrimination by SSR markers should be more effective than random amplified polymorphic DNA (RAPD) markers (Cipriani et al., 1996; Zhen et al., 2004). SSR markers exhibit simple banding patterns are codominant and highly repeatable among other advantages compared with RAPDs. Siblings or collections from bud mutations that share the majority of the

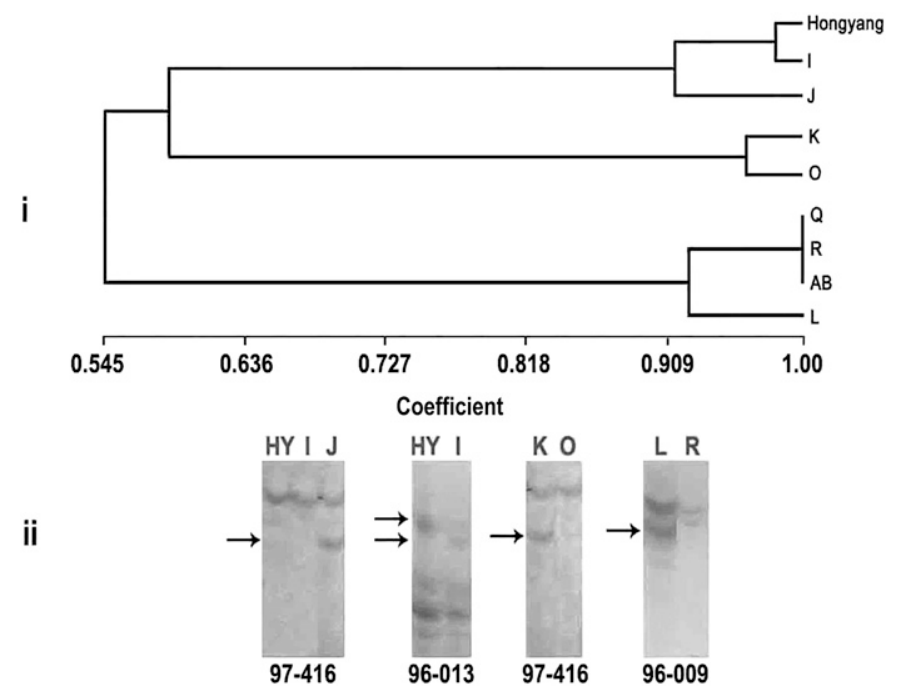

Fig. 4. UPGMA dendrogram using dice coefficient of genetic similarity among the indistinguishable accessions based on additional 28 SSRs. I, J, K, L, O, Q, R, and AB were codes of the accessions. Q, R, and $\mathrm{AB}$ were still not able to distinguish with each other (i). Allele difference on three loci $(97-416,96-$ 013, and 96-009) among 'Hongyang' (HY), I and J, and between 'Hongyang' and I, K and O, L and R, respectively, are shown (ii). UPGMA = unweighted pair group method with arithmetic averages; SSRs $=$ simple sequence repeats.

genome are always required for identification during kiwifruit breeding, which are unavoidably difficult in practice. For example, a natural bud mutation of 'Hayward' and 'Top Star' was not distinguishable from 'Hayward' using 25 RAPD primers (Cipriani et al., 1996), and a presumed half-sibling of 'Xiaya No. 1' from the same wild vine, 'Xiaya No. 15', also failed to be discerned from 'Xiaya No. 1' by eight SSR markers (Zhen et al., 2004). The 12 SSRs provided suitable ability to discriminate $\mathrm{H}$, 'Honghua', 'Hongmei'. and 'Chuhong' from each other. However, accessions C, I, J, AF, and 'Hongyang', $\mathrm{K}$ and $\mathrm{O}$ as well as R, L, Q, and $\mathrm{AB}$ failed to be discriminated using present SSRs. Therefore, variation in phenotypic characters had to be used for further diagnostics.

Among the first set of indistinguishable accessions (C, I, J, AF, and 'Hongyang'), average fruit weight of $\mathrm{J}$ was significantly higher than that of C, I, and AF (Table 2). Compared with C, J, AF, and 'Hongyang', I was detected to have fruits with higher total acids, lower SSC, and lower total sugar (Table 4). It was possible that $\mathrm{J}$ and I were different from each other and from each of $\mathrm{C}, \mathrm{AF}$, and 'Hongyang' at the genetic level. In the second set of indistinguishable accessions ( $R, L, Q$, and $A B)$, fruit size of $Q$ and $A B$ were notably smaller than that of $R$ and $L$ (Table 2). The inner pericarp red coloration of $\mathrm{L}, \mathrm{R}$, and $\mathrm{Q}$ was a deeper red than $\mathrm{AB}$ (Table 4). An increased number of SSRs should be screened and applied to evaluate more alleles and discriminate accessions based on genetic data. An additional 28 SSRs were adopted to discriminate the three sets of these accessions. It had been confirmed that a difference existed among I, J, and 'Hongyang', between $\mathrm{K}$ and $\mathrm{O}$, and between $\mathrm{L}$ and $\mathrm{Q}$ (R or AB) at the genetic level. Three selected SSRs
(97-416, 96-013, and 96-009) were effective for additional discrimination (Fig. 4).

In conclusion, several superior redfleshed accessions were shown to have bigger size, the most appealing visual coloration, higher SSC, and total sugar. Twelve pairs of SSR markers could be used to distinguish the limited number of accessions. The phenotypic data were still helpful for discriminating the indistinguishable accessions in this case. More accessions were confirmed to be genetically different based on an additional 28 SSRs. The results demonstrated that application of more SSR markers could improve the efficiency of identifying red-fleshed kiwifruit germplasm. Phenotypic and molecular characterization provides opportunities for enhancing curatorial management practices and promoting new selection for kiwifruit.

\section{Literature Cited}

Amodio, M.L., G. Colelli, J.K. Hasey, and A.A. Kader. 2007. A comparative study of composition and postharvest performance of organically and conventionally grown kiwifruits. J. Sci. Food Agr. 87:1228-1236.

Balestra, G.M., A. Mazzaglia, A. Quattrucci, M. Renzi, and A. Rossetti. 2009. Occurrence of Pseudomonas syringae pv. Actinidiae in Jin Tao kiwi plants in Italy. Phytopathol. Mediterr. 48:299-301.

Chen, Q.-L., Q.-H. Chen, X. Gu, G.-P. Shi, A.-C. Xu, Z.-Q. Qin, and S.-M. Wang. 2009. Achievements of kiwifruit breeding in China and our proposals in the future. China Fruits. 38:70-76.

Cipriani, G., R. Di Bella, and R. Testolin. 1996. Screening RAPD primers for molecular taxonomy and cultivar fingerprinting in the genus Actinidia. Euphytica 90:169-174.

Cui, Z.-X. 1993. Actinidia in China. Shandong Scientific Press, Jinan, Shandong. 
Doyle, J. and J. Doyle. 1987. A rapid DNA isolation procedure for small quantities of fresh leaf tissue. Phytochem. Bull. 19:11-15.

Feng, H. and H.-Z. Li. 2009. Epidemic law of Pseudomonas syringae pv. Actinidiae in kiwi plants in Zhouzhi County and its control using comprehensive methods. China Plant Protection. 29:28-30.

Ferguson, A.R. and H.W. Huang. 2007. Genetic resources of kiwifruit: Domestication and breeding. Hort. Rev. 33:1-121.

Ferguson, A.R. and A.G. Seal. 2008. Kiwifruit, p. 235-264. In: Hancock, J.F. (ed.). Temperate fruit crop breeding: Germplasm to genomics. Springer Science+Business Media B.V.

Hassall, A.K., G.J. Pringle, and E.A. Macrae. 1998. Development, maturation, and postharvest responses of Actinidia arguta (Sieb. Et Zucc.) Planch, ex Miq. fruit. New Zeal. J. Crop Hort. 26:95-108.

Hiratsuka, S., H. Onodera, Y. Kawai, T. Kubo, H. Itoh, and R. Wada. 2001. ABA and sugar effects on anthocyanin formation in grape berry cultured in vitro. Sci. Hort. 90:121-130.

Hopping, M.E. 1976. Structure and development of fruit and seeds in Chinese gooseberry (Actinidia chinensis Planch.). N.Z. J. Bot. 14:63-68.

Hopping, M.E. and E.M. Jerram. 1979. Pollination of kiwifruit (Actinidia chinensis Planch.): Stigma-style structure and pollen tube growth. N.Z. J. Bot. 17:233-240.

Howpage, D., V. Vithanage, and R. Spooner-Hart. 1998. Pollen tube distribution in the kiwifruit (Actinidia deliciosa A. Chev. C. F. Liang) pistil in relation to its reproductive process. Ann. Bot. 81:697-703.

Hu, Z.-R., W. Chen, and K.-M. Li. 2006. Descriptors and data standard for kiwifruit (Actinidia spp.). China Agriculture Press, Beijing, China.

Kawabata, S., Y. Kusuhara, Y. Li, and R. Sakiyama. 1999. The regulation of anthocyanin biosynthesis in Eustoma grandiflorum under low light conditions. J. Jpn. Soc. Hort. Sci. 68:519526.

Kelebek, H., S. Selli, A. Canbas, and T. Cabaroglu. 2009. HPLC determination of organic acids, sugars, phenolic compositions and antioxidant capacity of orange juice and orange wine made from a Turkish cv. Kozan. Microchem. J. 91:187-192.

Liang, C.-F. 1982. An addition to the infraspecific taxa of Actinidia chinensis Planch. Acta Phytotaxon. Sin. 20:101-104.
Liao, L. 2011. Landscape genetic structure and molecular phylogeography of Actinidia chinensis var. chinensis/deliciosa complex. Master diss., Wuhan Botanical Garden, Chinese Academy of Sciences, Wuhan, China.

Liu, Y., Y. Liu, and H. Huang. 2010. Genetic variation and natural hybridization among sympatric Actinidia species and the implications for introgression breeding of kiwifruit. Tree Genet. Genomes 6:801-813.

Mengoni, A., A. Gori, and M. Bazzicalupo. 2000 Use of RAPD and microsatellite (SSR) variation to assess genetic relationships among populations of Tetraploid alfafa, Medicago sativa. Plant Breed. 119:311-317.

Montefiori, M., R.V. Espley, D. Stevenson, J. Cooney, P.M. Datson, A. Saiz, R.G. Atkinson, R.P. Hellens, and A.C. Allan. 2010. Identification and characterization of F3GT1 and F3GGT1, two glycosyltransferases responsible for anthocyanin biosynthesis in red-fleshed kiwifruit (Actinidia chinensis). Plant J. 65:106118

Muggleston, S., M. McNeilage, R. Lowe, and H. Marsh. 1998. Breeding new kiwifruit cultivars The creation of Hort16A and Tomua. Orchardist New Zeal. 71:38-40.

Pirie, A. and M.G. Mullins. 1977. Interrelationship of sugars, anthocyanins, total phenols and dry weight in the skins of grape berries during ripening. Amer. J. Enol. Viticult. 28:204-209.

Pointer, M. R. 1981. A comparison of the CIE 1976 colour spaces. Color Res. Appl. 6:108-118.

Rashidi, M. and M. Gholami. 2008. Classification of fruit shape in kiwifruit using the analysis of geometrical attributes. American-Eurasian J. Agr. \& Environ. Sci. 3:258-263.

Rohlf, F.J. 2000. NTSYS-pc: Numerical taxonomy and multivariate analysis system, version 2.1 [CP/DK]. Exeter Ltd., Setauket, NY.

Rossetti, A. and G.M. Balestra. 2008. Pseudomonas syringae pv. Syringae on kiwifruit plants Epidemiological traits and its control, p. 65-68. In: Fatmi, M.B., et al. (eds.). Pseudomonas syringae pathovars and related pathogens. Springer Science+Business Media B.V.

Sanguinetti, C.J., E.D. Neto, and A.J.G. Simpson. 1994. Rapid silver staining and recovery of PCR products separated on polyacrylamide gels. Biotechniques 17:915-919.

Stintzing, F.C., A. Schieber, and R. Carle. 2003. Evaluation of color properties and chemical quality parameters of cactus juices. Eur. Food Res. Technol. 216:303-311.
UPOV. 2003. Protocol for distinctness, uniformity and stability tests Actinidia Lindl. (Kiwifruit). CPVO-TP/98/1, European Union, Community Plant Variety Office.

Wang, M.-Z. 2003. Sustainable breeding research of Actinidia chinensis var. rufopulpa. Resour. Develop. \& Market. 19:309-310.

Wang, M.-Z., X.-D. Li, Z.-S. Yu, M.-Z. Li, and S.-S. He. 2005a. Selection of a novel colorfulfleshed kiwifruit cultivar, 'Hongmei'. China Fruits. 4:7-9.

Wang, Z.-Y., C.-H. Zhong, and F.-W. Bu. 2005b. A new red-fleshed kiwifruit cultivar 'Chuhong'. Agr. Sci. \& Technol. 5:23-24.

Wang, M.-Z., W. Tang, and S.-X. Hou. 2006. Selection of a novel red-fleshed kiwifruit cultivar, 'Honghua'. China Fruits. 1:10-12.

White, J. 1986. Morphology of the fruit hairs in cultivars of Actinidia deliciosa var. deliciosa Actinidia eriantha and Actinidia rufa. N.Z. J. Bot. 24:415-423.

Wu, B.-L. 1992. Some new kiwifruit cultivars. J. Fruit Sci. 9:56-58

Wu, B.-L. and Z.-G. Wu. 1998. A new selection with superior quality and storage, 'Xinguan No. 2'. China Fruits. 4:29-30.

Wu, J.-H., A.R. Ferguson, B.G. Murray, Y. Jia P.M. Datson, and J. Zhang. 2011. Induced polyploidy dramatically increases the size and alters the shape of fruit in Actinidia chinensis. Ann. Bot. (Lond.) 109:169-179.

Zhang, L. 2010. Studies on characters segregation of progeny from three Actinidia intra/inter-specific crosses. PhD diss., Wuhan Botanical Garden, Chinese Academy of Sciences, Wuhan, China.

Zhen, Y., Z. Li, H. Huang, and Y. Wang. 2004 Molecular characterization of kiwifruit (Actinidia) cultivars and selections using SSR markers. J. Amer. Soc. Hort. Sci. 129:374382 .

Zhong, C.-H., Z.-Y. Wang, F.-W. Bu, and D.-F. Peng. 2007. Studies on the mechanism of red coloration in 'Chuhong' kiwifruit cultivar, $\mathrm{p}$. 213-218. In: Huang, H.-W. (ed.). Advance in actinidia research (IV). Science Press, Beijing, China

Zhu, D., G.S. Lawes, and I.L. Gordon. 2002 Estimates of genetic variability and heritability for vegetative and reproductive characters of kiwifruit (Actinidia deliciosa). Euphytica 124:93-98.

Zhu, H.-Y. and S.L. Li. 1993. A new kiwifruit cultivar, 'Huamei No. 2'. Shanxi Fruits 3:1213. 